\title{
Eco-digital Supply Chains through Blockchains
}

\author{
[ Pietro De Giovanni ]
}

\begin{abstract}
This paper shows how the implementation of Eco-digital Supply Chain can be realized through the adoption of a Blockchain technology. The focal company pushes suppliers at all tiers through the adoption of green practices for reducing the $\mathrm{CO}_{2}$ emissions and the related costs. Furthermore, logistics costs involved in the Supply Chain make the final assessment highly challenging. We identify the changes in both the Supply Chain structure and performance occurring when the Blockchain enables the creation of an Eco-digital Supply Chain. (Abstract)
\end{abstract}

Keywords -Eco-digital Supply Chain, Green Supply Chain, Blockchain, Environment, Networks (key words)

\section{Introduction(Heading 1)}

A Blockchain consists of a set of digital applications enabling firms and Supply Chains (SCs) to track, trace, and verify how goods transit and move along the supply chain, from the manufacturers to retailers through distribution centers and logistics facilities [1]. The information that firms share on a Blockchain serve to update, integrate, and monitor all information linked to transactions over the SC [2]. Today, SCs are globally dispersed, making the identification of SC members very difficult and, consequently, making impossible to follow their full set of actions [4]. Inefficiency over the SC can be linked to frauds and fake deals, cultural barriers and language differences, international regulations and legislative constraints, lack of trust and commitment of some suppliers call for additional information visibility, transparency, and verifiability to better manage and control the SC network, remove all potential business risks and mitigate the inefficient transactions characterizing the SC linkages [4], [6]. Blockchains should be adopted any time the network of relationships is not trustable since the parties hide critical information to the SC partners [7].

One key element that firms should consider is whether Blockchains also enable sustainable SCs. The latter is defined as the adoption of collaborative programs within the SC to make the forward and the backward processes greener and achieve social, environmental and economic targets [8]. Clearly, one big issue that SC partners experience nowadays is whether suppliers align the sustainable practices to the SC environmental targets, especially when firms work on online platforms [9].

Pietro De Giovanni (Author)

LUISS University

Rome, Italy

While traditional information like pricing, quantity and lead time can be known by the use of traditional SC tools like
Vendor Managed Inventory facilitating IBM Watson [3], other information linked to environmental performance, green practices, life cycle assessment and all sustainable information are not necessarily visible over the SC. In this sense, firms working in an SC can green the entire system only when the information about the production processes, the logistics and all life cycle assessment are visible. In addition, suppliers that are globally dispersed should receive ad hoc incentives to commit on environmental and social targets [11]. However, in a recent survey, [13] show that sustainability is a difficult target to reach in SCs, either in presence of incentives or in presence of services (e.g., [12]). Instead, the use of Blockchain can considerable help in this regard [14].

In this paper, we investigate the benefits that SCs have when moving from a traditional online platform that enables digital transformation to a Blockchain technology that enables an Eco-digital SC. The latter is defined as the adoption of digital tools to achieve social and environmental targets along with economic outcomes. The adoption of Blockchains can help in achieving both targets by requesting and then using all information needed to achieve an Ecodigital SC.

We start our analysis from a general SC network that involves four tiers: suppliers, depos, manufactures, and retailers. We solve two optimization problems, one linked to traditional online platforms and one using Blockchain technologies. Our results reveal that the use of Blockchain can considerably help SCs to increase both the operational efficiency and improve the environmental performance. However, this result can be achieved when the SC has a flexible structure within each tier, allowing the Blockchain to choose among several options such that the SC network optimizes both the logistics and the environmental costs. We propose an algorithm to carry out a traditional process of greening the SC. We show that Blockchain can allow firms to reach an Eco-digital SC in a faster and more efficiency way than traditional green collaborative practices that firms can undertake.

The remaining of the paper is structured as follows. Section II presents the SC networks that we seek to investigate. Section III details the data while Section IV introduces the results. Section V proposes a heuristic algorithm to green the SC and Section VI concludes.

\section{Supply Chain Networks}

In this paper, we assume that a Supply Chain (SC) is composed of four tiers, as it is displayed in Figure 1. We identify the Suppliers with $S$, the Depos with $D$, the Manufacturers with $M$, and the Retailers with $R$. Independent on the tier, firms send quantity of goods from their location to a destination, given by $q_{k j}$, with $k, j=S, D, M, R$. When goods are sent, each firm faces two costs: a logistics cost, $L$, and an environmental cost, $E$. When the SC takes decisions, the demand is stochastic and follows a normal distribution law $N \sim(4000,200)$. The SC 
has two possible configurations: traditional online platforms and Blockchain.

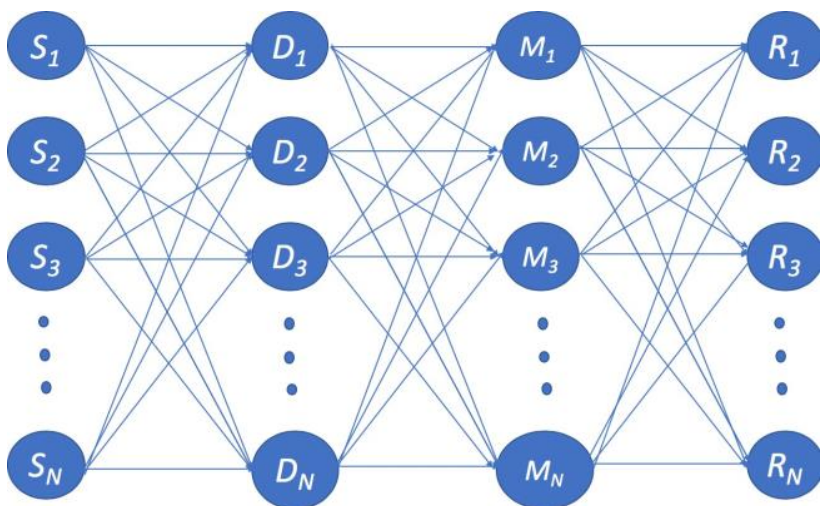

Figure 1. Supply Chain Network

When the SC uses traditional online platforms it is named Digital SC. The use of traditional online platforms is mainly linked to the low purchasing costs as well as by the efficient transportation mode of logistics providers. Therefore, the Digital SC achieves objectives of time, cost and quality without particular problems. Nevertheless, moving goods from on tier to another has several environmental consequences: the production of goods generates emissions and has harmful effects on the environment; the logistics system implies $\mathrm{CO}_{2}$ emissions, pollution and congestion; firms in each tier may use nonsustainable practices that can reflect into a low purchasing cost. Hence, the Digital SC sets strategies and makes firms moving goods over the chain by simply using the traditional optimization criteria of cost, time and quality.

In contrast, the Eco-Digital SC uses Blockchain technologies instead of traditional online platforms to make transactions over the chain. Because the Blockchain traces all information that decision makers want, it can verify the environmental performance of each SC member. By doing so, it ensures the visibility of both the logistics and the environmental performance and, consequently, the social transparency of all transactions. By identifying the underperforming SC members, the focal company can set some collaborative green programs to make the SC greener. However, some tradeoffs may considerably influence the firms' decisions since the couple logistics efficiency/environmental performance is not easy to be solved.

\section{A. Digital SC model}

Hereby we define a Digital SC model in which firms aim at minimizing the SC cost, which are linked to logistics and environmental costs. Both costs are proportional the activities linked to goods. Therefore, each good moved across the tiers generates a logistic cost and an environmental cost. The Digital SC solves the problem by only looking at the logistics efficiency. Therefore, the minimization problem is defined as follows:

$C=\min \left(q_{S D}, q_{D M}, q_{M R}\right)\left\{\Sigma_{S} \Sigma_{D} L_{S D} q_{S D}+\Sigma_{D} \Sigma_{M} L_{D M} q_{D M}+\Sigma_{M}\right.$

$\left.\Sigma_{R} L_{M R} q_{M R}\right\}$

s.t.:

'S, $\Sigma_{D} q_{S D} \geq \max$ Capacity(S)

\section{'D, $\Sigma_{M} q_{D M} \geq \max \operatorname{Capacity}(D)$}

"M, $\Sigma_{R} q_{M R} \geq \max \operatorname{Capacity}(M)$

"S, $\Sigma_{D} q_{S D} \geq \operatorname{Demand}(S)$

"D, $\Sigma_{M} q_{M R} \geq \operatorname{Demand}(D)$

"M, $\Sigma_{R} q_{M R} \geq \operatorname{Demand}(R)$

"D, $\operatorname{Capacity}(D)=\Sigma_{S} q_{S D}$.

"M, $\operatorname{Capacity}(M)=\Sigma_{D} q_{D M}$

'R, $\operatorname{Capacity}(R)=\Sigma_{M} q_{M R}$

"S, "D, "M, "R, $q_{k j} \geq 0$

$C_{F}=\Sigma_{S} \Sigma_{D}\left(E_{S D}+L_{S D}\right) q_{S D}+\Sigma_{D} \Sigma_{M}\left(E_{D M}+L_{D M}\right) q_{D M}$

$$
\left.+\Sigma_{M} \Sigma_{R}\left(E_{M R}+L_{M R}\right) q_{M R}\right\}
$$

with $C_{F}$ being the final Digital SC costs outcome.

\section{B. Eco-Digital SC}

In this section, we propose the cost minimization problem that the Eco-digital SC solves by taking into consideration both the logistics costs and the environment issues. Therefore, the firms consider both elements when setting their optimal strategies. The problem is as follows:

$$
\begin{gathered}
C_{F}=C=\min \left(q_{S D}, q_{D M}, q_{M R}\left\{\Sigma_{S} \Sigma_{D}\left(E_{S D}+L_{S D}\right) q_{S D}+\Sigma_{D} \Sigma_{M}\right.\right. \\
\left.\left(E_{D M}+L_{D M}\right) q_{D M}+\Sigma_{M} \Sigma_{R}\left(E_{M R}+L_{M R}\right) q_{M R}\right\}
\end{gathered}
$$

s.t.: contraints in Eqs. (2)-(11).

\section{Data and methodology}

To highlight the benefits that Blockchain can generate in terms of Eco-digital SC, we move away from the general SC configurations displayed in Figure 1 and focus on a particular case as displayed in Figure 2, consisting of 10 suppliers, 3 depos, 1 manufacturer, and 4 retailers.

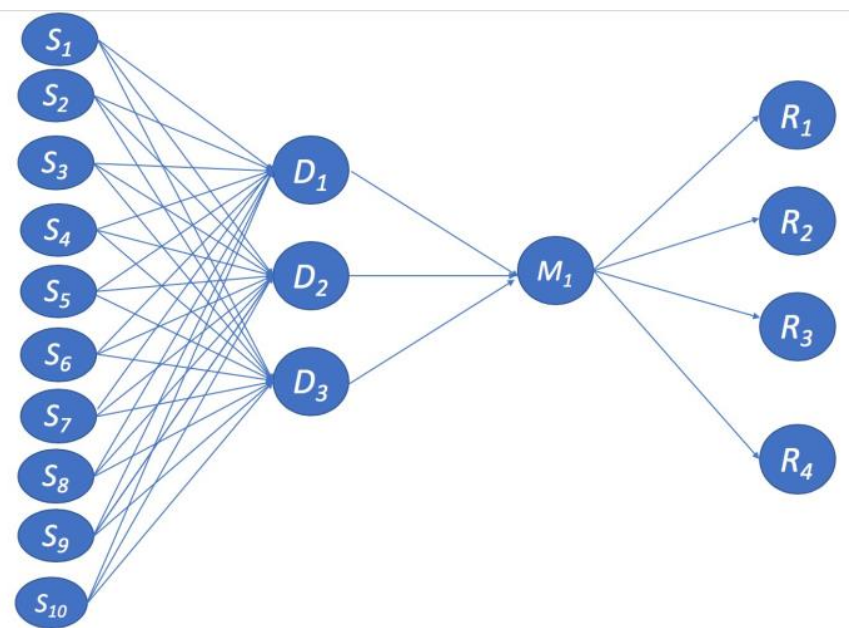

Figure 2. Supply Chain Network to optimize

All the data relatively to the marginal logistics cost and the marginal environmental cost have been obtained through simulations. All costs are assumed to be Uniform distributed according to different law and depending on the tier. In fact, each tier implies different complexity, which links to the number of operations and environmental implications. 
Specifically, the simulation design follows the procedure described in [9] and consists of the following distributions for logistics cost, $L$, and environmental cost, $E$ :

- Tier S-D's logistics and environmental costs are distributed as $U\left(L_{S D}\right) \sim(0.1,0.5) \quad$ and $U\left(E_{S D}\right) \sim(0.05,1)$, respectively;

- Tier D-M' logistics and environmental costs are distributed as $U\left(L_{D M}\right) \sim(0.4,0.8) \quad$ and $U\left(E_{D M}\right) \sim(0.5,2.5)$, respectively;

- Tier M-R's logistics and environmental costs are distributed as $U\left(L_{M R}\right) \sim(0.1,1)$ and $U\left(E_{M R}\right) \sim(0.1,1)$, respectively;

The suppliers send raw material to the depos at a marginal cost $L_{S D}$ and each movement generates a marginal cost per $\mathrm{CO}_{2}$ emissions $E_{S D}$. The full cost analysis is displayed in Table 1.

\begin{tabular}{|l|c|c|c|c|c|c|}
\hline \multirow{2}{*}{} & \multicolumn{2}{|c|}{ Depo $_{1}$} & \multicolumn{2}{c|}{ Depo $_{2}$} & \multicolumn{2}{c|}{ Depo $_{3}$} \\
\cline { 2 - 7 } & $\boldsymbol{E}_{\boldsymbol{S D}}$ & $\boldsymbol{L}_{\boldsymbol{S D}}$ & $\boldsymbol{E}_{\boldsymbol{S D}}$ & $\boldsymbol{L}_{\boldsymbol{S D}}$ & $\boldsymbol{E}_{\boldsymbol{S D}}$ & $\boldsymbol{L}_{\boldsymbol{S D}}$ \\
\hline $\boldsymbol{S}_{\boldsymbol{1}}$ & 0,7 & 0,1 & 0,3 & 0,1 & 0,3 & 0,3 \\
\hline $\boldsymbol{S}_{\boldsymbol{2}}$ & 0,2 & 0,2 & 0,2 & 0,2 & 0,2 & 0,2 \\
\hline $\boldsymbol{S}_{3}$ & 0,2 & 0,2 & 0,2 & 0,2 & 0,1 & 0,1 \\
\hline $\boldsymbol{S}_{\boldsymbol{4}}$ & 0,3 & 0,3 & 0,3 & 0,3 & 0,9 & 0,1 \\
\hline $\boldsymbol{S}_{\mathbf{5}}$ & 0,8 & 0,15 & 0,3 & 0,3 & 0,3 & 0,3 \\
\hline $\boldsymbol{S}_{\boldsymbol{6}}$ & 0,2 & 0,2 & 0,2 & 0,2 & 0,2 & 0,2 \\
\hline $\boldsymbol{S}_{\mathbf{7}}$ & 0,3 & 0,3 & 0,3 & 0,3 & 0,1 & 0,1 \\
\hline $\boldsymbol{S}_{\boldsymbol{8}}$ & 0,8 & 0,1 & 0,3 & 0,3 & 0,9 & 0,1 \\
\hline $\boldsymbol{S}_{9}$ & 0,2 & 0,2 & 0,2 & 0,2 & 0,2 & 0,2 \\
\hline $\boldsymbol{S}_{10}$ & 0,2 & 0,2 & 0,2 & 0,2 & 0,1 & 0,1 \\
\hline
\end{tabular}

Table 1. Marginal logistics and environmental cost between tiers S-D.

When the depos manage the raw materials and make some consolidation operations, the entire process requires internal logistics efforts as well as a strong external logistics network to manage big bulks. Consider that the up stream of the chain is composed of several small suppliers, sending their goods to the depos. Then, depos need to organize the bulks and manage the logistics through a different network of transport modes. Therefore, the logistics cost $L_{D M}$ represents the logistics cost of transferring some goods from each depo to the manufacturer's plant, while $E_{D M}$ is the environmental cost generated by all activities within these two tiers. Table 2 displays the full cost analysis linked to the links between depos and manufacturer.

Finally, the manufacturer makes several operations including: decomposition of bulks, quality control, machine setup, transformation, packaging and consolidation. Then, it transfers bulks of finished products to the retailer to be sold. Overall, the manufacturer faces costs relatively to all operations that it carries out, $L_{M R}$, as well as environmental costs generated by all processes that it activates, $E_{M R}$. All data are displayed in Table 3.

\section{Manufacturer}

\begin{tabular}{|l|c|c|}
\hline \multicolumn{1}{l|}{} & $\boldsymbol{E}_{\boldsymbol{D M}}$ & $\boldsymbol{L}_{\boldsymbol{D M}}$ \\
\hline Depo $_{1}$ & 2 & 0,5 \\
\hline Depo $_{2}$ & 1,3 & 0,6 \\
\hline Depo $_{3}$ & 0,8 & 0,7 \\
\hline
\end{tabular}

Table 2. Marginal logistics and environmental cost between tiers D-M.

\begin{tabular}{|l|l|l|l|l|l|l|l|l|}
\cline { 2 - 9 } & \multicolumn{2}{c|}{ Retailer $_{1}$} & \multicolumn{2}{l|}{ Retailer $_{2}$} & \multicolumn{2}{l|}{ Retailer $_{3}$} & \multicolumn{2}{l|}{ Retailer $_{4}$} \\
\cline { 2 - 9 } & $\boldsymbol{E}_{M R}$ & $\mathbf{L}_{M R}$ & $\boldsymbol{E}_{M R}$ & $\mathbf{L}_{M R}$ & $\boldsymbol{E}_{M R}$ & $\boldsymbol{L}_{M R}$ & $\boldsymbol{E}_{M R}$ & $\boldsymbol{L}_{M R}$ \\
\hline Manufact. & 1 & 0,2 & 0,7 & 0,4 & 0,4 & 0,6 & 0,2 & 0,5 \\
\hline
\end{tabular}

Table 3. Marginal logistics and environmental cost between tiers $\mathrm{M}-\mathrm{R}$.

We assume that all suppliers have supply capacity of 3.000 units per day, the depos have an inventory management of 10.000 units per day, and the manufacturer has a production capacity of 25.000 units per day. The retailers sell products to four different markets, which are located quite far from each other. Accordingly, consumers cannot travel from a place to another to buy the same good since it would be very expensive.

\section{Results}

We display the results of the OR model in Figure 1.

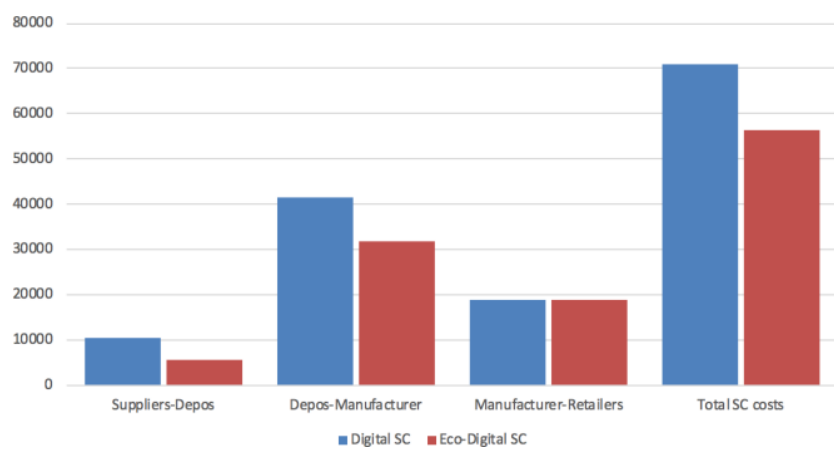

Figure 3. Cost comparison.

Accordingly, we can leave the following managerial insights. The use of Blockchain to implement an Eco-Digital SC is economically worthwhile as it allows the overall Supply Chain cost to decrease of $20 \%$. Therefore, the theoretical advantages of implementing a Blockchain should be compared to the real economic benefits that are dictated by a comprehensive cost analysis. In most of the cases, IT developers and consultants present the Blockchain technology as the solution to all issues of transparency and visibility. While this is correct there are also some weaknesses that firms should careful take into consideration. First, the implementation of a Blockchain implies some initial investments costs, e.g., cost for implementing the Blockchain solution and integrating it within the current ERP that firms use. Second, firms seeking to implement a Blockchain should make training to the SC partners to use this technology and depart from the use of traditional systems. Third, the SCs should investigate on the impact of Blockchains on the decisional lead time. Blockchain 
requires the approval of all stakeholders linked to transactions and the decisions making process are postponed if the consensus is not reached.

Overall, the convenience of implementing a Blockchain should be compared to its advantages. In fact, the implementation and the use of a Blockchain should not be more costly than $20 \%$ benefits that the SC obtains. In such a case, although the economic advantages can be minimal, the additional soft benefits are still granted to the SC and should also be taken into consideration.

Our results reveal that the convenience of adopting a Blockchain for Eco-digital purposes is mainly linked to the supply risks emerging from the SC structure. First, the upstream SC management is highly diversified because several suppliers live in the supply park and they have different performance. In fact, the couple logistics/environmental costs and a Pareto analysis reveal that the tier Suppliers-Depos can be highly improved since there are only a few suppliers of category AA. The latter are suppliers A for logistics costs and A for the environmental impact, while most of the remaining suppliers are of category $\mathrm{BA}$ or $\mathrm{AB}$. Therefore, there are options to make the supply chain shifting suppliers according to the combination of logistics and environmental costs as well as their impact on the SC efficiency, which consists of around 33\% improvement.

In the same vein, the tier Depos-Manufacturer can considerably impact on the SC total cost. This is mainly due to the presence of multiple Depos having different performance in terms of logistics costs and environmental impact. Consider that the tier Depos-Manufacturer accounts for around $66 \%$ of the overall SC efficiency. Therefore, SC should be designed to guarantee flexibility in the supplier selection since the use of Blockchain can be extremely helpful to decrease the emissions in an efficient way.

The previous statements are clearly confirmed by the results in the tier Manufacturer-Retailers. Hereby, the SC costs are independent of the implementation of Blockchain technologies. This result mainly depends on the high SC risks occurring at the Manufacturer level. The Retailers, in fact, do not have any alternative way to carry out their business than working with one sole Manufacturer. Accordingly, the Blockchain enables the Eco-Digital SCs if and only if the SC structure is sufficiently flexible and decisions makers can adjust the SC structure according to the targets that the SC has. Therefore, anytime the SC is not flexible and the supply risks are very high, the use of Blockchains does not bring full advantages.

Our analysis, in fact, informs that the SC structure changes when moving from a Digital SC to an Eco-Digital SC implemented through Blockchains. In the former case, the Suppliers being part of the SC business are S1, S2, S3, S5, S6, S7, and S8. In the latter case the SC consists of the following suppliers: S1, S2, S3, S6, S7, S9 and S10. Instead, when analyzing the tier Manufacturer-Retailers, the structure is the same since capacity constraints influence the SC optimization problem that cannot be solved otherwise. Finally, the tier Depos-Manufacturer is quasi flexible since the structure is highly rigid, but the Depos have unused capacity so that the SC can play with the different allocation solutions to find the best optimal. The shadow price analysis reveals that Depo 3 has the best impact on the total
SC cost; therefore, it is the most convenient Depo as it has a marginal impact on the logistics costs and a low environmental impact as well. The SC should eventually converge the maximum flow to Depo 3 and then chose the rest. Also, if the SC has a budget to invest, it should definitely reinforce the Depo 3's capacity.

Finally, the use of Blockchain allows the decision maker to optimize the SC by also accounting for the real environmental performance of all SC members involved. This information is most likely hidden when working with traditional digital platforms, where suppliers mainly provide information on quantity and pricing. The outcomes linked to Blockchains can be used to make the SC greener through the adoption of collaborative programs.

\section{v. Green Collaboration through a heuristic algorithm}

In this section, we move forward and use the results of the Blockchain analysis to make the SC greener. The adoption of Blockchain entails significant environmental improvements and logistics savings, considering that we estimate an overall improvement of $20 \%$, where $15 \%$ links to environmental benefits and $5 \%$ links to logistics savings. One additional effort that the SC can do consists of the implementation of sustainable collaborative programs to make some suppliers greener. In this regard, we propose a heuristic algorithm with the objective of improving the SC results. The algorithm is composed of the following steps:

Step 1. Use the solution given by solving the Eco-Digital SC problem through Blockchain;

Step 2. Identify "brown" suppliers and make them green;

Step 3. Solve the optimization problem and estimate the budget needed to run such collaborative programs;

Step 4. Do steps 2 and 3 till improvements are no longer possible.

By using this algorithm, we obtain the results as displayed in Figures 4 and 5.

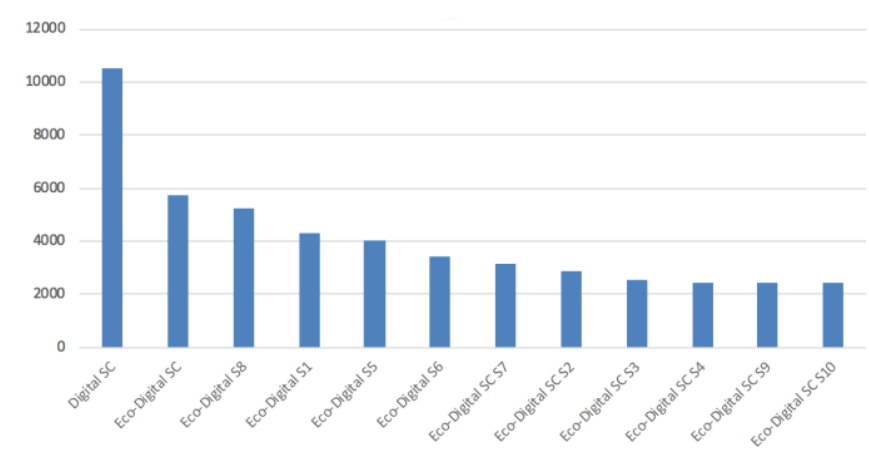

Figure 4. Eco-digital SC transformation and supply costs 


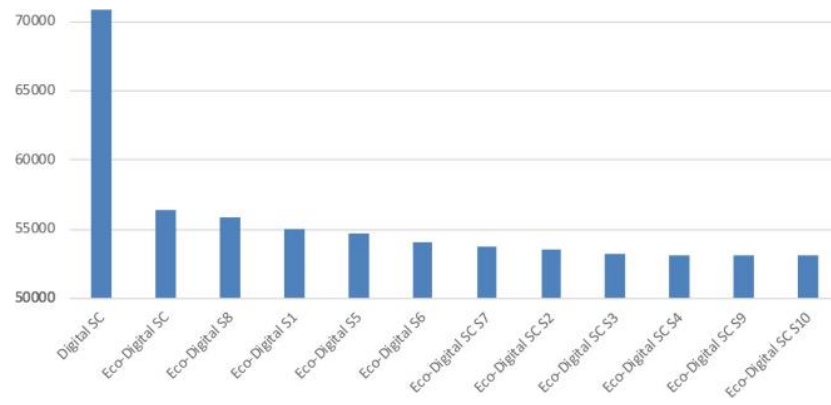

Figure 5. Eco-digital SC transformation and total costs

Our findings inform us that the Blockchain allow firms to green the SC and achieve the Eco-digital target. Interestingly, firms have two options to green the SC: either adopt Blockchains or green the supply activities of all supply partners. In the former, the SC benefits of $20 \%$ supply cost reduction; in the latter, it gains cost savings for $6 \%$. This result opens new managerial insights in the literature of SC management aiming at green targets. The traditional practices like adopting green raw material, green energy, sustainable programs, limit the use of toxic substances, recycling programs, life cycle assessment, reverse logistics programs (see [10] for a comprehensive list) seem to be less effective nowadays. This is probably due to historical pressures that firms have on social and environmental issues, for which firms have acquired multiple competences and gained experience on managing green processes. The adoption of Blockchains for Ecodigital purposes is a new challenge and its implementation can bring considerable environmental and cost saving advantages.

This result is also confirmed by the analysis of the total SC cost displayed in Figure 5. Accordingly, Blockchains can bring a substantial decrease of environmental concerns and cost inefficiency. Rather, greening suppliers results in a suboptimal policy since the benefits disappear after some points. That is, after the strategic suppliers are greened, spending additional budgets for greening other suppliers does not result in significant improvements. In this sense, the Blockchain turns out to be much more effective since it optimizes the global SC system in one instance, resulting highly appealing and promising.

\section{vi. Conclusions}

This paper compares two SC structures: Digital SC and Eco-digital SC. The former carries out transactions in traditional online platforms in which only a few information is available for SC members (e.g., price and quantity). The latter uses Blockchains to finalize transactions, resulting in an optimization tools that allows firms to simultaneously consider traditional operations along with environmental issues. Accordingly, Blockchain enables Eco-digital SCs.

Our results demonstrate that the adoption of Blockchains provides considerable cost saving benefits. The optimization tools behind the Blockchain allows firms to gain transparency and visibility on several aspects of the business. Clearly, if SC members are not willing to share information on the Blockchain, they are withdrawn from the SC. This step becomes very critical because SCs can enjoy the advantages of Blockchains only when the tiers present multiple options. That is, if the Blockchain can flexibly select partners inside each tier, Eco-digital SC can easily be achieved. Therefore, Blockchain enables Eco-digital transformations only when the SC structure is flexible. Our findings, in fact, show that the Blockchain works very well in the upstream where the supplier park is composed of ten suppliers; hence, the Blockchain can select the best supplier according to the information published on the Blockchain platform. In contrast, the tier linked to the manufacturer presents only one firm. In such a case, the Blockchain cannot do much since there is only one alternative to carry out the SC business. Then, Blockchain should be followed by diversification options inside each tier and flexible SC structure. Finally, our findings demonstrate that the environmental benefits of Blockchains are wider than the environmental benefits that firms would obtain by greening the supply process of each single supplier. Therefore, Blockchains are a real option to guarantee high environmental performance and cost benefits at the same time, then achieving an Eco-digital SC.

This research is not free of limitations, which are suggested here to inspire future research in the same domain. The SC that we propose focuses on logistics and environmental costs. Other costs and operational problems can be taken into consideration like inventory, quality and service. The SC network model is evaluated in a static manner, while future research can look at the dynamic version of this model. We used simulated data to run our analysis. Future research can collect primary data or use clinical cases to validate our model. Industry 4.0 technologies can be used and complement Blockchain technologies to pursue Eco-digital SC targets. This is an ongoing research projects that the author seeks to develop.

\section{References}

[1] Cole, R., Stevenson, M., \& Aitken, J. (2019). Blockchain technology: implications for operations and supply chain management. Supply Chain Management: An International Journal, 24(4), 469-483.

[2] De Giovanni, P. (2019a). Blockchains in Supply Chain Management and Smart Contracts. WP010920

[3] De Giovanni, P. (2019b). Artificial Intelligence in a Dynamic Supply Chain with VMI. WP010925

[4] Saberi, S., Kouhizadeh, M., \& Sarkis, J. (2019). Blockchains and the supply chain: Findings from a broad study of practitioners. IEEE Engineering Management Review.

[5] Liu B. \& De Giovanni, P. (2020). Green Process Innovation with Industry 4.0 technologies and Supply Chain Coordination, Annals of Operations Research, to appear.

[6] Dolgui, A., Ivanov, D., Potryasaev, S., Sokolov, B., Ivanova, M., \& Werner, F. (2019). Blockchain-oriented dynamic modelling of smart contract design and execution in the supply chain. International Journal of Production Research, 1-16.

[7] Chakrabarti, A., \& Chaudhuri, A. K. (2017). Blockchain and its scope in retail. International Research Journal of Engineering and Technology, 4(7), 3053-3056.

[8] Ramani, V., \& De Giovanni, P. (2017). A two-period model of product cannibalization in an atypical Closed-loop Supply Chain with endogenous returns: The case of DellReconnect. European Journal of Operational Research, 262(3), 1009-1027.

[9] De Giovanni, P., Karray, S., \& Martín-Herrán, G. (2019). Vendor Management Inventory with consignment contracts and the benefits of cooperative advertising. European Journal of Operational Research, 272(2), 465-480. 
[10] Cariola A. \& De Giovanni, P. (2020). Process Innovation with Industry 4.0, lean practices and environmental performance. Research In Transportation Economics, to appear.

[11] Genc, T. S., \& De Giovanni, P. (2018). Optimal return and rebate mechanism in a closed-loop supply chain game. European Journal of Operational Research, 269(2), 661-681.

[12] De Giovanni, P., \& Ramani, V. (2018). Product cannibalization and the effect of a service strategy. Journal of the Operational Research Society, 69(3), 340-357.

[13] De Giovanni, P., \& Zaccour, G. (2019). A selective survey of gametheoretic models of closed-loop supply chains. 4OR, 17(1), 1-44.

[14] Kshetri, N. (2018). 1 Blockchain's roles in meeting key supply chain management objectives. International Journal of Information Management, 39, 80-89. 